20010019022

NASA/CR-2000-210632

ICASE Report No. 2000-49

NASA-CR-2000-210632

20010029126

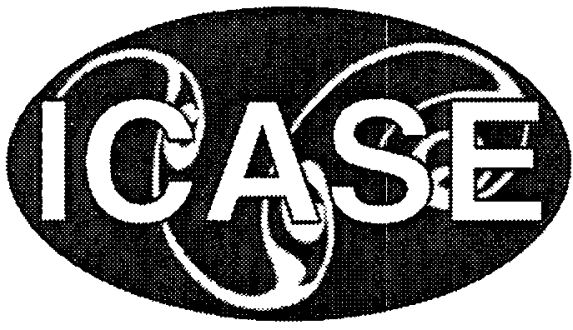

(

\title{
A Technique of Treating Negative Weights in WENO Schemes
}

Jing Shl, Changqing Hu, and Chi-Wang Shu

Brown University, Providence, Rhode Island

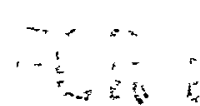

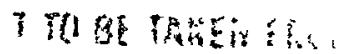

$\sqrt{31} 252$

December 2000 


\section{The NASA STI Program Office ... in Profile}

Since its founding, NASA has been dedicated to the advancement of aeronautics and space science The NASA Scientific and Technical Information (STI) Program Office plays a key part in helping NASA maintain this important role

The NASA STI Program Office is operated by Langley Research Center, the lead center for NASA's scientific and technical information The NASA STI Program Office provides access to the NASA STI Database, the largest collection of aeronautical and space science STI in the world The Program Office is also NASA's institutional mechanısm for disseminating the results of its research and development actıvities These results are published by NASA in the NASA STI Report Series, which includes the following report types

- TECHNICAL PUBLICATION Reports of completed research or a major significant phase of research that present the results of NASA programs and include extensive data or theoretical analysis Includes compilations of significant scientific and technical data and information deemed to be of continuing reference value NASA's counterpart of peer-reviewed formal professional papers, but having less stringent limitations on manuscript length and extent of graphic presentatıons

\section{- TECHNICAL MEMORANDUM} Scientific and technical findings that are prelımınary or of specialızed interest, e g , quick release reports, workıng papers, and biblıgraphies that contain mınımal annotatıon Does not contain extensive analysis

- CONTRACTOR REPORT Scientific and technical findings by NASA-sponsored contractors and grantees
- CONFERENCE PUBLICATIONS Collected papers from scientific and technical conferences, symposia, seminars, or other meetıngs sponsored or cosponsored by NASA

- SPECIAL PUBLICATION Scientıfic, technical, or historical information from NASA programs, projects, and missions, often concerned with subjects having substantial public interest

- TECHNICAL TRANSLATION Englishlanguage translations of foreign scientific and technical material pertinent to NASA's mission

Specialızed services that complement the STI Program Office's diverse offerings include creatıng custom thesaurı, bulding customızed data bases, organızıng and publıshing research results even providing videos

For more information about the NASA STI Program Office, see the following

- Access the NASA STI Program Home Page at http://www.sti.nasa.gov

- Email your question via the Internet to help@stı nasa gov

- Fax your question to the NASA STI Help Desk at (301) 621-0134

- Telephone the NASA STI Help Desk at (301) 621-0390

- Write to NASA STI Help Desk NASA Center for AeroSpace Information 7121 Standard Drive Hanover, MD 21076-1320 


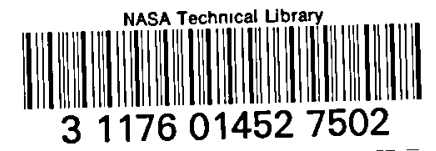

NASA/CR-2000-210632

ICASE Report No. 2000-49

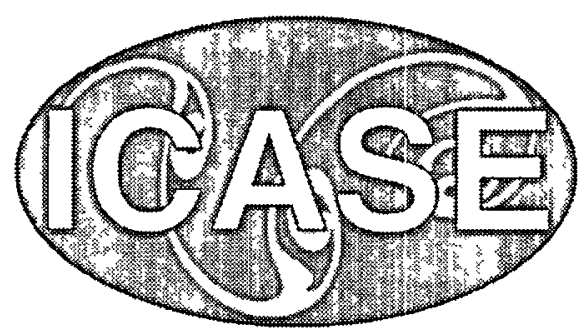

\section{A Technique of Treating Negative Weights in WENO Schemes}

Jing Shl, Changqing Hu, and Cht-Wang Shu

Brown University, Providence, Rhode Island

ICASE

NASA Langley Research Center

Hampton, Virginia

Operated by Universities Space Research Association

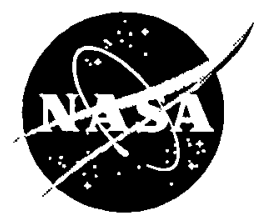

National Aeronautics and Space Administration

Langley Research Center

Hampton, Virgınıa 23681-2199
Prepared for Langley Research Center under Contract NAS1-97046

December 2000 
Avallable from the following

NASA Center for AeroSpace Information (CASI)

7121 Standard Drve

Hanover, MD 21076-1320

(301) 621-0390
Natıonal Technical Information Service (NTIS) 5285 Port Royal Road

Springfield. VA 22161-2171

(703) 487-4650 


\title{
A TECHNIQUE OF TREATING NEGATIVE WEIGHTS IN WENO SCHEMES*
}

\author{
JING SHI ${ }^{\dagger}$, CHANGQING HU ${ }^{\ddagger}$ AND CHI-WANG SHU
}

\begin{abstract}
High order accurate weighted essentially non-oscillatory (WENO) schemes have recently been developed for finite difference and finte volume methods both in structured and in unstructured meshes A key idea in WENO scheme is a linear combination of lower order fluxes or reconstructions to obtain a higher order approximation The combination coefficients, also called linear weights, are determined by local geometry of the mesh and order of accuracy and may become negative WENO procedures cannot be applied directly to obtain a stable scheme if negative linear weights are present Previous strategy for handling this difficulty is by either regrouping of stencils or reducing the order of accuracy to get rid of the negatıve linear weights In this paper we present a sımple and effectıve technique for handling negatıve linear weights without a need to get rid of them Test cases are shown to illustrate the stability and accuracy of this approach
\end{abstract}

Key words. weıghted essentially non-oscillatory, negatıve weıghts, stabılıty, high order accuracy, shock calculation

Subject classification. Applied and Numerical Mathematıcs

1. Introduction. High order accurate werghted essentially non-oscllatory (WENO) schemes have recently been developed to solve a hyperbolic conservation law

$$
u_{t}+\nabla \quad f(u)=0
$$

The first WENO scheme was constructed in [18] for a thrd order finite volume version in one space dimension In [10], third and fifth order finite difference WENO schemes in multı space dimensions are constructed, with a general framework for the design of the smoothness indicators and nonlinear weights Later, second, third and fourth order finite volume WENO schemes for 2D general triangulation have been developed in [4] and [8] Very high order finte difference WENO schemes (for orders between 7 and 13) have been developed in [1] Central WENO schemes have been developed in [12], [13] and [14]

WENO schemes are designed based on the successful ENO schemes in [7, 23, 24] Both ENO and WENO use the idea of adaptıve stencis in the reconstruction procedure based on the local smoothness of the numerical solution to automatically achieve high order accuracy and non-oscillatory property near discontinuities ENO uses just one (optimal in some sense) out of many candidate stencils when doing the reconstruction, while WENO uses a convex combination of all the candidate stencils, each being assigned a nonlınear weight which depends on the local smoothness of the numerical solution based on that stencil WENO improves upon ENO in robustness, better smoothness of fluxes, better steady state convergence, better provable convergence propertıes, and more efficiency For a detaled review of ENO and WENO schemes, we refer to the lecture notes $[21,22]$

*Research supported by ARO grants DAAG55-97-1-0318 and DAAD19-00-1-0405, NSF grants DMS-9804985 and ECS9906606, NASA Langley grant NAG-1-2070 and Contract NAS1-97046 while the third author was in resıdence at ICASE, NASA Langley Research Center, Hampton, VA 23681-2199, and AFOSR grant F49620-99-1-0077

†Dıvision of Applied Mathematics, Brown University, Providence, RI 02912 E-mall shı@cfm brown edu

$\ddagger$ Division of Applied Mathematics, Brown University, Providence, RI 02912 Current address Oracle, 3OP6, 500 Oracle Parkway, Redwood Shores, CA 94065 E-mal phu@us oracle com

$\S$ Division of Applied Mathematics, Brown University, Providence, RI 02912 E-mail shu@cfm brown edu 
WENO schemes have already been widely used in applications Some of the examples include dynamical response of a stellar atmosphere to pressure perturbations [3], shock vortex interactions and other gas dynamics problems [5], [6], incompressible flow problems [26], Hamilton-Jacobı equations [9], magnetohydrodynamics [11], underwater blast-wave focusing [15], the composite schemes and shallow water equations [16], [17], real gas computations [19], wave propagation using Fey's method of transport [20], etc

A key idea in WENO schemes is a lnear combination of lower order fluxes or reconstructions to obtain a higher order approximation The combination coefficients, also called linear weights, are determmed by local geometry of the mesh and order of accuracy and may become negative WENO procedures cannot be applied directly to obtain a stable scheme if negative linear weights are present Previous strategy for handling this difficulty is by either regrouping of stencils (e g in [8]) or reducing the order of accuracy (e $g$ in [12]) to get rid of the negatıve linear weights In this paper we present a simple and effectıve technique for handling negative linear weights without a need to get rid of them Test cases will be shown to llustrate the stability and accuracy of this approach

We first summarize the general WENO reconstruction procedure, consisting of the following steps We assume we have a given cell $\Delta$ (which could be an interval in $1 \mathrm{D}$, a rectangle in a $2 \mathrm{D}$ tensor product mesh, or a triangle in a 2D unstructured mesh) and a fixed point $x^{G}$ withm or on one edge of the cell

1 We identify several stencils $\mathcal{S}_{\jmath}, \jmath=1,, q$, such that $\Delta$ belongs to each stencil We denote by $\mathcal{T}=\bigcup_{j=1}^{q} \mathcal{S}_{j}$ the larger stencll which contains all the cells from the $q$ stencils

2 We have a (relatively) lower order reconstruction or interpolation function (usually a polynomial), denoted by $p_{\jmath}(x)$, associated with each of the stencils $\mathcal{S}_{\jmath}$, for $\jmath=1, q$ We also have a (relatively) higher order reconstruction or interpolation function (again usually a polynomial), denoted by $Q(x)$, associated with the larger stencl $\mathcal{T}$

3 We find the combination coefficients, also called linear weights, denoted by $\gamma_{1}, \quad, \gamma_{q}$, such that

$$
Q\left(x^{G}\right)=\sum_{\jmath=1}^{q} \gamma_{\jmath} p_{\jmath}\left(x^{G}\right)
$$

for all possible given data in the stencls These linear weights depend on the mesh geometry, the point $x^{G}$, and the specific reconstruction or interpolation requirements, but not on the given solution data in the stencils

4 We compute the smoothness indicator, denoted by $\beta_{\jmath}$, for each stencll $\mathcal{S}_{\jmath}$, which measures how smooth the function $p_{J}(x)$ is in the target cell $\triangle$ The smaller this smoothness indicator $\beta_{J}$, the smoother the function $p_{J}(x)$ is in the target cell In all of the current WENO schemes we are using the following smoothness indicator

$$
\beta_{\jmath}=\sum_{1 \leq|\alpha| \leq h} \int_{\Delta}|\Delta|^{|\alpha|-1}\left(D^{\alpha} p_{\jmath}(x)\right)^{2} d x
$$

for $\jmath=1, \quad, q$, where $k$ is the degree of the polynomial $p_{\jmath}(x)$ and $|\Delta|$ is the area of the cell $\triangle$ in $2 \mathrm{D}$ This factor is different for $1 \mathrm{D}$ or $3 \mathrm{D}$ the purpose of it is to bring the smoothness indicator invariant under spatial scalıng

5 We compute the nonlınear weights based on the smoothness indicators

$$
\omega_{\jmath}=\frac{\tilde{\omega}_{\jmath}}{\sum_{\jmath} \tilde{\omega}_{\jmath}}, \quad \tilde{\omega}_{\jmath}=\frac{\gamma_{\jmath}}{\left(\varepsilon+\beta_{\jmath}\right)^{2}}
$$


where $\gamma_{\jmath}$ are the linear weıghts determined in step 3 above, and $\varepsilon$ is a small number to avold the denominator to become 0 We are using $\varepsilon=10^{-6}$ in all the computations in this paper The final WENO approximation or reconstruction is then given by

$$
R\left(x^{G}\right)=\sum_{\jmath=1}^{q} \omega_{\jmath} p_{\jmath}\left(x^{G}\right)
$$

We remark that all the coefficients in the above steps which depend on the mesh but not on the data of the numerical solution, should be computed and stored at the beginning of the code after the generation of the mesh but before the time evolution starts

We now use a simple example to lllustrate the steps outlined above We assume we are given a uniform mesh $I_{\imath}=\left(x_{\imath-1 / 2}, x_{\imath+1 / 2}\right)$ and cell averages of a function $u(x)$ in these cells, denoted by $\bar{u}_{\imath}$ We would like to find a fifth order WENO reconstruction to the point value $u\left(x_{\imath+1 / 2}\right)$, based on a stencil of five cells $\left\{I_{\imath-2}, I_{\imath-1}, I_{\imath}, I_{\imath+1}, I_{\imath+2}\right\}$, with the target cell containing the point $x_{\imath+1 / 2}$ chosen as $\triangle=I_{\imath}$

In step 1 above we could have the following three stencls

$$
\mathcal{S}_{1}=\left\{I_{\imath-2}, I_{\imath-1}, I_{\imath}\right\}, \quad \mathcal{S}_{2}=\left\{I_{\imath-1}, I_{\imath}, I_{\imath+1}\right\}, \quad \mathcal{S}_{3}=\left\{I_{\imath}, I_{\imath+1}, I_{\imath+2}\right\},
$$

which make up a larger stencil

$$
\mathcal{T}=\left\{I_{\imath-2}, I_{2-1}, I_{2}, I_{\imath+1}, I_{\imath+2}\right\}
$$

In step 2 above we would have three polynomals $p_{\jmath}(x)$ of degree at most two, with their cell averages agreeing with that of the function $u$ in the three cells in each stencll $\mathcal{S}_{J}$ The higher order function $Q(x)$ is a polynomial of degree at most four, with its cell averages agreeing with that of the function $u$ in the five cells in the larger stencll $\mathcal{T}$ The three lower order approximations to $u\left(x_{i+1 / 2}\right)$, associated with $p_{J}(x)$, in terms of the given cell averages of $u$, are given by

$$
\begin{aligned}
& p_{1}\left(x_{\imath+1 / 2}\right)=\frac{1}{3} \bar{u}_{\imath-2}-\frac{7}{6} \bar{u}_{\imath-1}+\frac{11}{6} \bar{u}_{\imath}, \\
& p_{2}\left(x_{\imath+1 / 2}\right)=-\frac{1}{6} \bar{u}_{\imath-1}+\frac{5}{6} \bar{u}_{\imath}+\frac{1}{3} \bar{u}_{\imath+1}, \\
& p_{3}\left(x_{\imath+1 / 2}\right)=\frac{1}{3} \bar{u}_{\imath}+\frac{5}{6} \bar{u}_{\imath+1}-\frac{1}{6} \bar{u}_{\imath+2}
\end{aligned}
$$

Each of them is a third order approximation to $u\left(x_{\imath+1 / 2}\right)$ The higher order approximation to $u\left(x_{\imath+1 / 2}\right)$, associated with $Q(x)$, is given by

$$
Q\left(x_{\imath+1 / 2}\right)=\frac{1}{30} \bar{u}_{\imath-2}-\frac{13}{60} \bar{u}_{\imath-1}+\frac{47}{60} \bar{u}_{\imath}+\frac{9}{20} \bar{u}_{\imath+1}-\frac{1}{20} \bar{u}_{\imath+2}
$$

which is a fifth order approximation to $u\left(x_{\imath+1 / 2}\right)$

In step 3 above we would have

$$
\gamma_{1}=\frac{1}{10}, \quad \gamma_{2}=\frac{3}{5}, \quad \gamma_{3}=\frac{3}{10}
$$

It can be readily verified, using (1 6) and (1 7), that

$$
Q\left(x_{\imath+1 / 2}\right)=\gamma_{1} p_{1}\left(x_{\imath+1 / 2}\right)+\gamma_{2} p_{2}\left(x_{\imath+1 / 2}\right)+\gamma_{3} p_{3}\left(x_{\imath+1 / 2}\right)
$$

for all possible given data $\bar{u}_{\jmath}, \jmath=\imath-2, \imath-1, \imath, \imath+1, \imath+2$ 

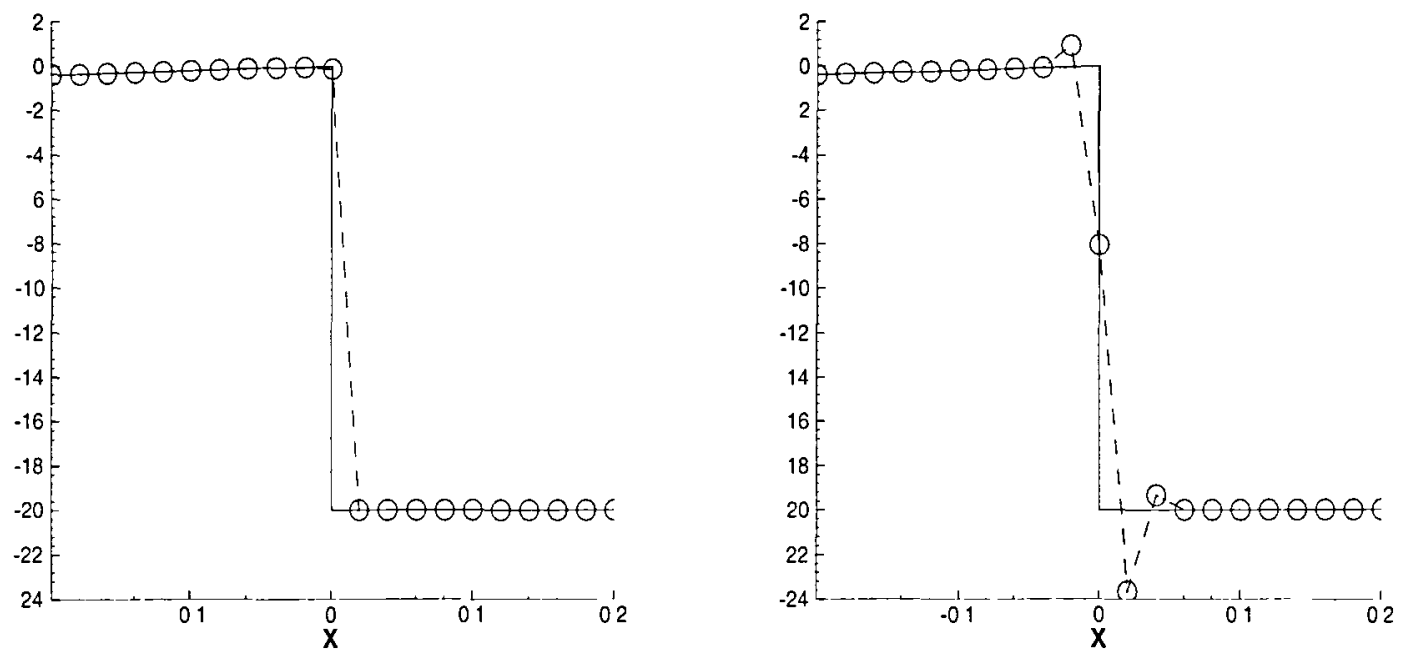

Fig 11 Reconstructions to $u\left(x_{\imath+1 / 2}\right)$ Solid lines exact function, symbols numerical approximations Left fifth order WENO Right fifth order traditional

In step 4 above we could easily work out from (13) the three smoothness indicators given by

$$
\begin{aligned}
& \beta_{1}=\frac{13}{12}\left(\bar{u}_{\imath-2}-2 \bar{u}_{\imath-1}+\bar{u}_{\imath}\right)^{2}+\frac{1}{4}\left(\bar{u}_{\imath-2}-4 \bar{u}_{\imath-1}+3 \bar{u}_{\imath}\right)^{2}, \\
& \beta_{2}=\frac{13}{12}\left(\bar{u}_{\imath-1}-2 \bar{u}_{\imath}+\bar{u}_{\imath+1}\right)^{2}+\frac{1}{4}\left(\bar{u}_{\imath-1}-\bar{u}_{\imath+1}\right)^{2}, \\
& \beta_{3}=\frac{13}{12}\left(\bar{u}_{\imath}-2 \bar{u}_{\imath+1}+\bar{u}_{\imath+2}\right)^{2}+\frac{1}{4}\left(3 \bar{u}_{\imath}-4 \bar{u}_{\imath+1}+\bar{u}_{\imath+2}\right)^{2}
\end{aligned}
$$

We notice in particular that the linear weights $\gamma_{1}, \gamma_{2}, \gamma_{3}$ in step 3 above are all positive In such cases, the WENO reconstruction procedure outlined above and the scheme based on it work very well In Fig 11 we plot the approximation to $u(x)$ for a discontinuous function $u(x)=2 x$ for $x \leq 0$ and $u(x)=-20$ otherwise, by the fifth order WENO reconstruction on the left and by the fifth order traditional reconstruction (1 7 ) on the right, with a mesh $x_{\imath}=(\imath-04965) \Delta x$ with $\Delta x=002$ We can clearly see that WENO avolds the over and undershoots near the discontinuity

We now look at another simple example where some of the linear weights in step 3 above would become negative We have exactly the same setting as above except now we seek the reconstruction not at the cell boundary but at the cell center $x_{\imath}$ This is needed by the central schemes with staggered grids [12] Thus, step 1 would stay the same as above, step 2 would produce

$$
\begin{aligned}
& p_{1}\left(x_{\imath}\right)=-\frac{1}{24} \bar{u}_{\imath-2}+\frac{1}{12} \bar{u}_{\imath-1}+\frac{23}{24} \bar{u}_{\imath}, \\
& p_{2}\left(x_{\imath}\right)=-\frac{1}{24} \bar{u}_{\imath-1}+\frac{13}{12} \bar{u}_{\imath}-\frac{1}{24} \bar{u}_{\imath+1}, \\
& p_{3}\left(x_{\imath}\right)=\frac{23}{24} \bar{u}_{\imath}+\frac{1}{12} \bar{u}_{\imath+1}-\frac{1}{24} \bar{u}_{\imath+2}
\end{aligned}
$$

Each of them is a third order reconstruction to $u\left(x_{\imath}\right)$ The higher order reconstruction to $u\left(x_{\imath}\right)$, associated with $Q(x)$, is given by

$$
Q\left(x_{\imath}\right)=\frac{3}{640} \bar{u}_{\imath-2}-\frac{29}{480} \bar{u}_{\imath-1}+\frac{1067}{960} \bar{u}_{\imath}-\frac{29}{480} \bar{u}_{\imath+1}+\frac{3}{640} \bar{u}_{\imath+2},
$$



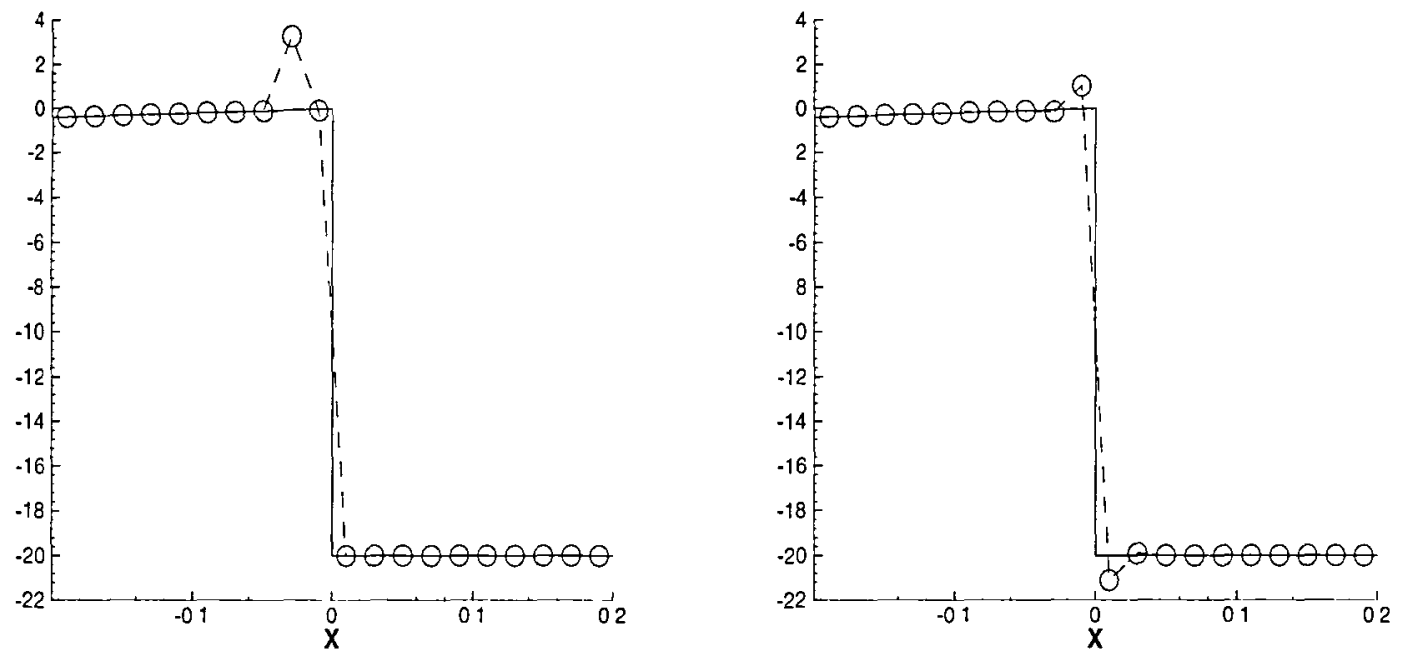

FIG 12 Reconstructions to $u\left(x_{\imath}\right)$ Soldd lines exact function, symbols numerical approximations Left fifth order WENO Right fifth order traditional

which is a fifth order reconstruction to $u\left(x_{\imath}\right)$ Step 3 would produce the following weights

$$
\gamma_{1}=-\frac{9}{80}, \quad \gamma_{2}=\frac{49}{40}, \quad \gamma_{3}=-\frac{9}{80}
$$

Notice that two of them are negative The smoothness indicators in step 4 will remain the same This time, the WENO approximation, shown at the left of Fig 12, is less satisfactory (in fact, even worse than a traditional fifth order reconstruction show on the right), because of the negative linear weights

We remark that negative linear weights do not appear in finite difference WENO schemes in any spatial dimensions for conservation laws for any order of accuracy [10], [1], and they do not appear in one dimensional as well as some mult1-dimensional finte volume WENO schemes for conservation laws Unfortunately, ther do appear in some other cases, such as the central WENO schemes using staggered meshes we have seen above, high order finite volume schemes for two dimensions described in [8] and in this paper, and finte difference WENO approximations for second derivatives

While on approximation alone the appearance of negatıve linear weights might be annoying but perhaps not fatal (Fig 12 ), in solving a PDE the result might be more serıous As an example, in Fig 13 we shon the results of using a fourth order finite volume WENO scheme [8] on a non-uniform triangular mesh shown at the left, which has negative linear weights, for solving the two dimensional Burgers equation

$$
u_{t}+\left(\frac{u^{2}}{2}\right)_{x}+\left(\frac{u^{2}}{2}\right)_{y}=0
$$

In the domain $[-2,2] \times[-2,2]$ with an initial condition $u_{0}(x, y)=03+07 \sin \left(\frac{\pi}{2}(x+y)\right)$ and periodic boundary conditions We can see that serious oscillation appears in the numerical solution once the shock has developed The oscillation eventually leads to instability and blowing up of the numerical solution for this example

The main purpose of this paper is to develop a simple and effectıve technique for handling negative linear werghts without a need to get rid of them Test cases will be shown to illustrate the stability and accuracy of this approach 

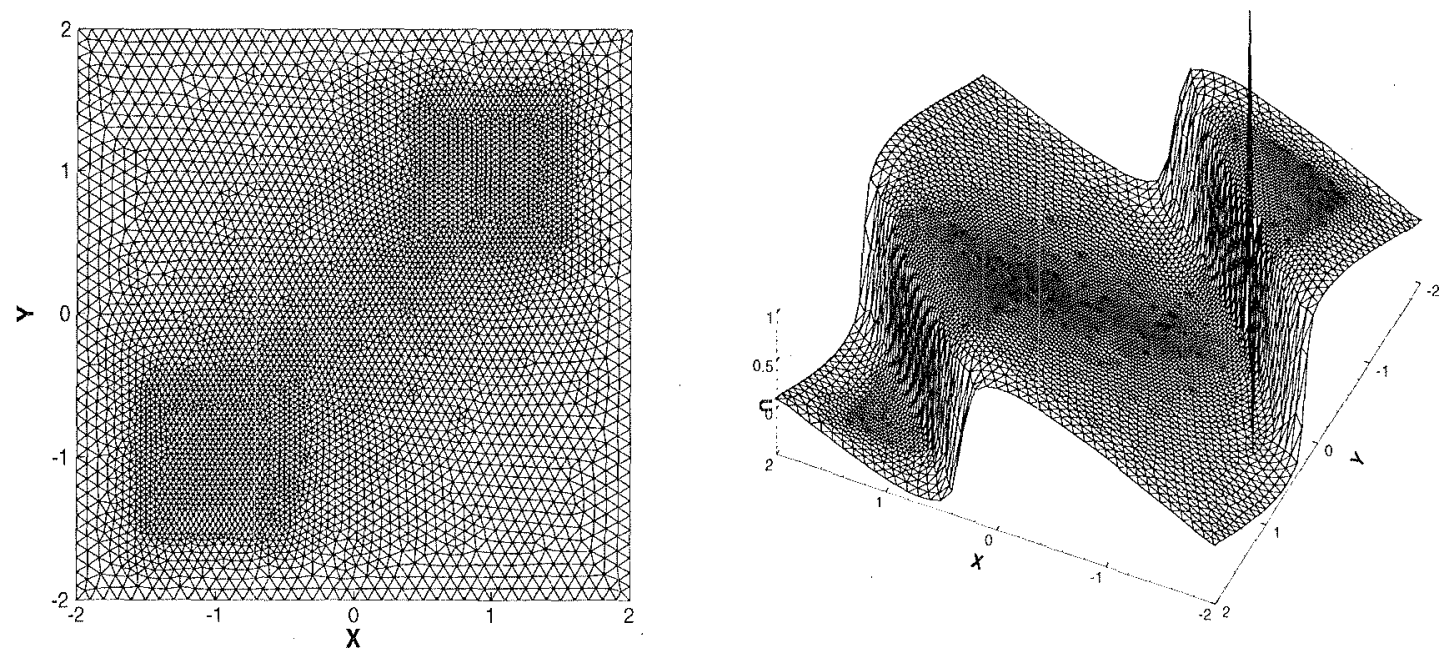

FIG. 1.3. 2D Burgers' equation. Left: non-uniform triangular mesh used in the computation. Right: fourth order WENO result at $t=0.473$, cfl $=0.2$, without any special treatment for the negative linear weights.

2. A splitting technique. We now introduce a splitting technique to treat the negative weights. It is very simple, involves little additional cost, yet is quite effective. The WENO procedure outlined in the previous section is only modified in step 5 in the following way:

5 If $\min \left(\gamma_{1}, \ldots, \gamma_{q}\right) \geq 0$ proceed as before. Otherwise, we split the linear weights into two parts: positive and negative. Define

$$
\tilde{\gamma}_{i}^{+}=\frac{1}{2}\left(\gamma_{i}+\theta\left|\gamma_{i}\right|\right), \quad \tilde{\gamma}_{i}^{-}=\tilde{\gamma}_{i}^{+}-\gamma_{i}, \quad i=1, \ldots, q
$$

where we take $\theta=3$ all the numerical tests. We then scale them by

$$
\sigma^{ \pm}=\sum_{j=1}^{q} \tilde{\gamma}_{j}^{ \pm} ; \quad \gamma_{i}^{ \pm}=\tilde{\gamma}_{i}^{ \pm} / \sigma^{ \pm}, \quad i=1, \ldots, q .
$$

We now have two split polynomials

$$
Q^{ \pm}\left(x^{G}\right)=\sum_{j=1}^{q} \gamma_{j}^{ \pm} p_{j}\left(x^{G}\right)
$$

which satisfy

$$
Q\left(x^{G}\right)=\sigma^{+} Q^{+}\left(x^{G}\right)-\sigma^{-} Q^{-}\left(x^{G}\right) .
$$

We can then define the nonlinear weights (1.4) for the positive and negative groups $\gamma_{j}^{\ddagger}$ separately, denoted by $\omega_{j}^{ \pm}$, based on the same smoothness indicator $\beta_{j}$. We will then define the WENO approximation $R^{ \pm}\left(x^{G}\right)$ separately by (1.5), using $\omega_{j}^{ \pm}$, and form the final WENO approximation by

$$
R\left(x^{G}\right)=\sigma^{+} R^{+}\left(x^{G}\right)-\sigma^{-} R^{-}\left(x^{G}\right) .
$$

We remark that the key idea of this decomposition is to make sure that every stencil has a significant representation in both the positive and the negative weight groups. Within each group, the WENO idea of 

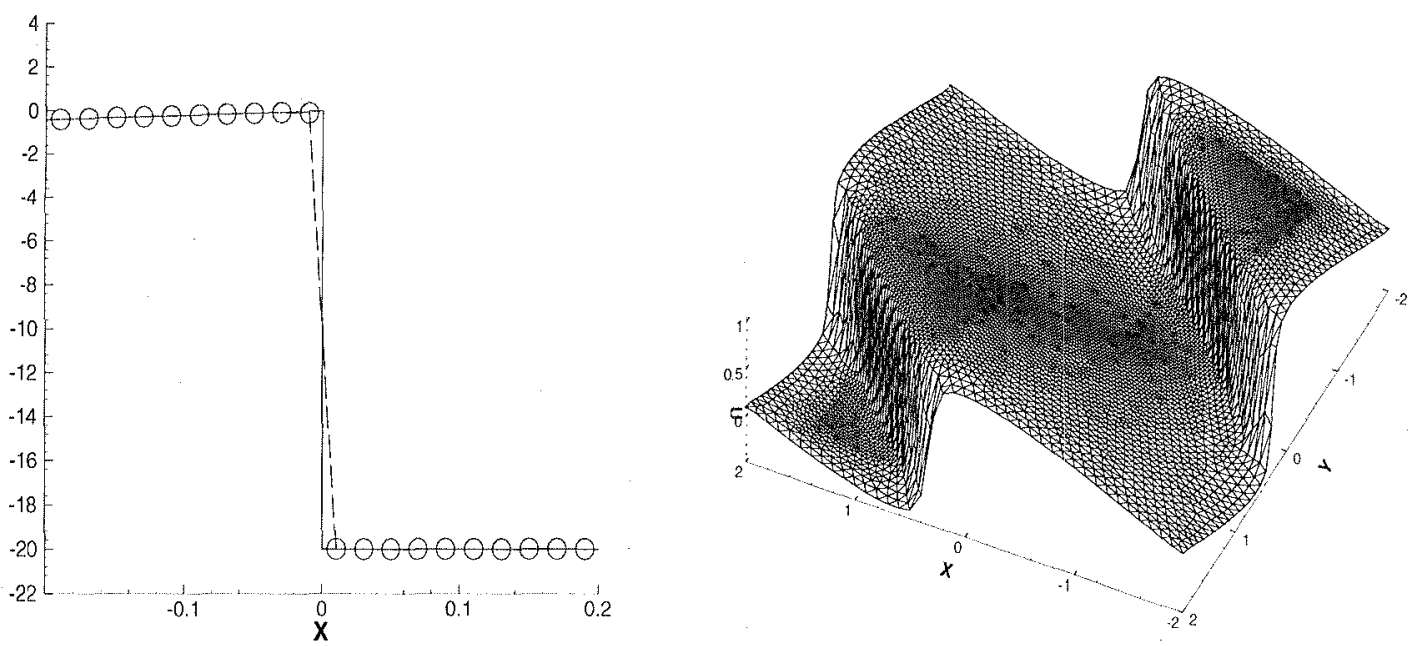

FIG. 2.1. WENO approximations with the splitting treatment for negative linear weights. Left: approximation to u( $\left.x_{i}\right)$. Right: Burgers equation, solution at $t=5 / \pi^{2}$, cf $=0.2$.

redistributing the weights subject to a fixed sum according to the smoothness of the approximation is still followed as before.

For the simple example of fifth order WENO reconstruction to $u\left(x_{i}\right)$, the split linear weights corresponding to (2.1) are, before the scaling,

$$
\tilde{\gamma}_{1}^{+}=\frac{9}{80}, \quad \tilde{\gamma}_{1}^{-}=\frac{9}{40}, \quad \tilde{\gamma}_{2}^{+}=\frac{49}{20}, \quad \tilde{\gamma}_{2}^{-}=\frac{49}{40}, \quad \tilde{\gamma}_{3}^{+}=\frac{9}{80}, \quad \tilde{\gamma}_{3}^{-}=\frac{9}{40} .
$$

We notice that, as the most expensive part of the WENO procedure, namely the computation of the smoothness indicators (1.3), has not changed, the extra cost of this positive/negative weight splitting is very small.

However this simple and inexpensive change makes a big difference to the computations. In Fig. 2.1 we show the result of the two previous unsatisfactory cases, the fifth order WENO reconstruction to $u\left(x_{i}\right)$ in Fig. 1.2 left, and the approximation to the Burgers equation in Fig. 1.3 right, now using WENO schemes with this splitting treatment. We can see clearly that the results are now as good as one would get from WENO schemes having only positive linear weights.

It is easy to prove that the splitting maintains the accuracy of the approximation in smooth regions. We will demonstrate this fact in the following sections. We will also demonstrate the effectiveness of this simple splitting technique through a few selected numerical examples in the next sections. The main WENO schemes we will consider are fifth order finite volume WENO schemes on Cartesian meshes, and the third and fourth order finite volume WENO schemes on triangular meshes. In both cases negative linear weights appear regularly.

The calculations are performed on SUN Ultra workstations and also on the IBM SP parallel computer at TCASCV of Brown University. The parallel efficiency of the method is excellent (more than 90\%).

\section{2D finite volume WENO schemes on Cartesian meshes.}

3.1. The schemes. We describe two different ways to construct fifth order finite volume WENO schemes on Cartesian meshes. Comparing with finite difference WENO methods [10], finite volume meth- 
ods have the advantage of an applicability of using arbitrary non-uniform meshes, at the price of increased computational cost [2]

We define the cell

$$
I_{\imath, J} \equiv\left[x_{\imath-\frac{1}{2}}, x_{\imath+\frac{1}{2}}\right] \times\left[y_{\jmath-\frac{1}{2}}, y_{j+\frac{1}{2}}\right]
$$

for $\imath=1, \quad, m, \jmath=1, \quad, n$, Where $I_{\imath, \jmath}$ needs not be uniform or smooth varying

The three-point Gaussian quadrature rule is used at each cell edge when evaluating the numerical flux In order to maintain fifth order accuracy Let $\left(x^{G}, y^{G}\right)$ denote one of the Gaussian quadrature points at the cell boundary of $I_{\imath, J}$ given by $\Gamma \equiv\left\{x=x_{\imath-\frac{1}{2}}, y_{\jmath-\frac{1}{2}} \leq y \leq y_{\jmath+\frac{1}{2}}\right\}$ There are two ways to perform a WENO reconstruction at the point $\left(x^{G}, y^{G}\right)$

Genuine 2D The first WENO reconstruction is genuine 2D finite volume We can see that there are totally nine stencils $\mathcal{S}_{s, t}(s, t=-1,0,1)$ Each stencil $\mathcal{S}_{s, t}$ contans $3 \times 3$ cells centered around $I_{l+s, j+t}$ On each stencil we can construct a $Q^{2}$ polynomial (tensor product of second order polynomials in $x$ and $y$ ) satısfying the cell average condition ( 1 e 1 its cell average in each cell inside the stencil equals to the given value) Let $\mathcal{T}=\bigcup_{s, t=-1}^{1} \mathcal{S}_{s, t}$, which contans $5 \times 5$ cells centered around $I_{\imath, J}$ On $\mathcal{T}$ we can construct a $Q^{4}$ polynomial satisfying the cell average condition The WENO reconstruction is then performed according to the steps outlined in sections 1 and 2

We would like to make the following remarks

1 By using a Lagrange interpolation basis, we can easily find the unique linear weights

2 Even for a uniform mesh, a negative linear weight appears for the middle Gaussian point $\left(x^{G}, y^{G}\right)=$ $\left(x_{\imath-\frac{1}{2}}, y_{J}\right)$ Such appearance of negative linear weights has also been observed in the central WENO schemes [12], see the example in sections 1 and 2 before

3 By Taylor expansions, we can prove that the smoothness indicators yield a uniform fifth order accuracy in smooth regions See [10] for the method of proof

Dimension by Dımension The second WENO reconstruction exploits the tensor product nature of the interpolation we use This WENO procedure is performed on a dimension by dimension fashion The WENO schemes applied in [5], [6] belong to this class Consider the point $\left(x^{G}, y^{G}\right)$ as above First we perform a one dimensional WENO reconstruction in the $y$ direction, in order to get the one dimensional cell averages (in the $x$ direction) $w\left(\bullet, y^{G}\right)$ Then we perform another one dimensional WENO reconstruction to $w$ in the $x$ direction, to obtain the final reconstructed point value at $\left(x^{G}, y^{G}\right)$

We would like to make the following remarks

1 For a scalar equation, the underlying linear reconstructions of the above two versions are equivalent For nonlinear WENO reconstructions they are not equivalent Both of them are fifth order accurate but the actual errors on the same mesh may be different, see Table 31 below

2 For systems of conservation laws such as the Euler equations of gas dynamics, both versions of the WENO reconstruction should be performed in local characteristic fields

3 The dimension by dimension version of the WENO reconstruction is less expensive and requires smaller memory than the genume two dimensional version The CPU time saving is about a factor of 4 for the Euler equations in our implementation The computed results are mostly simlar from both versions

In the following, we will give numerical examples computed by the above WENO schemes Splitting technique has been used in all the computations when negatıve linear weights appear We will show the 
TABLE 31

$2 D$ vortex evolution

\begin{tabular}{|c|c|c|c|c|c|}
\hline & & genuin & FV & dım-by & $\mathrm{hm}$ \\
\hline $\mathrm{N}$ & $\Delta x$ & $L^{\infty}$ error & order & $L^{\infty}$ error & order \\
\hline 20 & $671 \mathrm{E}-1$ & $438 \mathrm{E}-2$ & & $526 \mathrm{E}-2$ & \\
\hline 40 & $377 \mathrm{E}-1$ & 3 10E-3 & 459 & $566 \mathrm{E}-3$ & 386 \\
\hline 80 & $201 \mathrm{E}-1$ & $120 \mathrm{E}-4$ & 515 & $396 \mathrm{E}-4$ & 422 \\
\hline 160 & $100 \mathrm{E}-1$ & $439 \mathrm{E}-6$ & 476 & $796 \mathrm{E}-6$ & 562 \\
\hline 320 & 5 00E-2 & $188 \mathrm{E}-7$ & 453 & $290 \mathrm{E}-7$ & 477 \\
\hline
\end{tabular}

results for both smooth and discontinuous problems

3.2. 2D vortex evolution. First, we check the accuracy of the WENO schemes constructed above The two dimensional vortex evolution problem [21], [8] is used as a test problem

We solve the Euler equations for compressible flow in $2 \mathrm{D}$

$$
U_{t}+f(U)_{x}+g(U)_{y}=0
$$

where

$$
\begin{gathered}
U=(\rho, \rho u, \rho v, E)^{T} \\
f(U)=\left(\rho u, \rho u^{2}+p, \rho u v, u(E+p)\right)^{T}, \\
g(U)=\left(\rho v, \rho u v, \rho v^{2}+p, v(E+p)\right)^{T}
\end{gathered}
$$

Here $\rho$ is the density, $(u, v)$ is the velocity, $E$ is the total energy, $p$ is the pressure, related to the total energy by $E=\frac{p}{\gamma-1}+\frac{1}{2} \rho\left(u^{2}+v^{2}\right)$ with $\gamma=14$

The setup of the problem is the mean flow is $\rho=1, p=1,(u, v)=(1,1)$ and the computational domain is $[0,10] \times[0,10]$ We add, to the mean flow, an isentropic vortex (perturbations in $(u, v)$ and the temperature $T=\frac{p}{\rho}$, no perturbation in the entropy $S=\frac{p}{\rho^{\gamma}}$ )

$$
(\delta u, \delta v)=\frac{\epsilon}{2 \pi} e^{05\left(1-r^{2}\right)}(-\bar{y}, \bar{x}), \quad \delta T=-\frac{(\gamma-1) \epsilon^{2}}{8 \gamma \pi^{2}} e^{1-r^{2}}, \quad \delta S=0
$$

where $(\bar{x}, \bar{y})=(x-5, y-5), r^{2}=\bar{x}^{2}+\bar{y}^{2}$, and the vortex strength $\epsilon=5$

We use non-uniform meshes which are obtained by an independent random shifting of each point from a uniform mesh in each direction withn $30 \%$ of the mesh sizes The solution is computed up to $t=2$ Table 31 shows the $L^{\infty}$ errors of $\rho$ We can see that both the genuine two dimensional finite volume WENO scheme and the dimension by dimension finite volume WENO scheme can acheve the desired order of accuracy whlle the genuine two dimensional scheme gives smaller errors for the same mesh

3.3. Oblique shock tubes. The purpose for this test is to see the capability of the rectangular WENO schemes in resolving waves that are oblique to the computational meshes For detalls of the problem, we refer to [10] The 2D Sod's shock tube problem is solved where the initial jump makes an angle $\theta$ against the $x$ axis We take our computational domain to be $[0,6] \times[0,1]$ and the intral jump starting at $(x, y)=(225,0)$ 

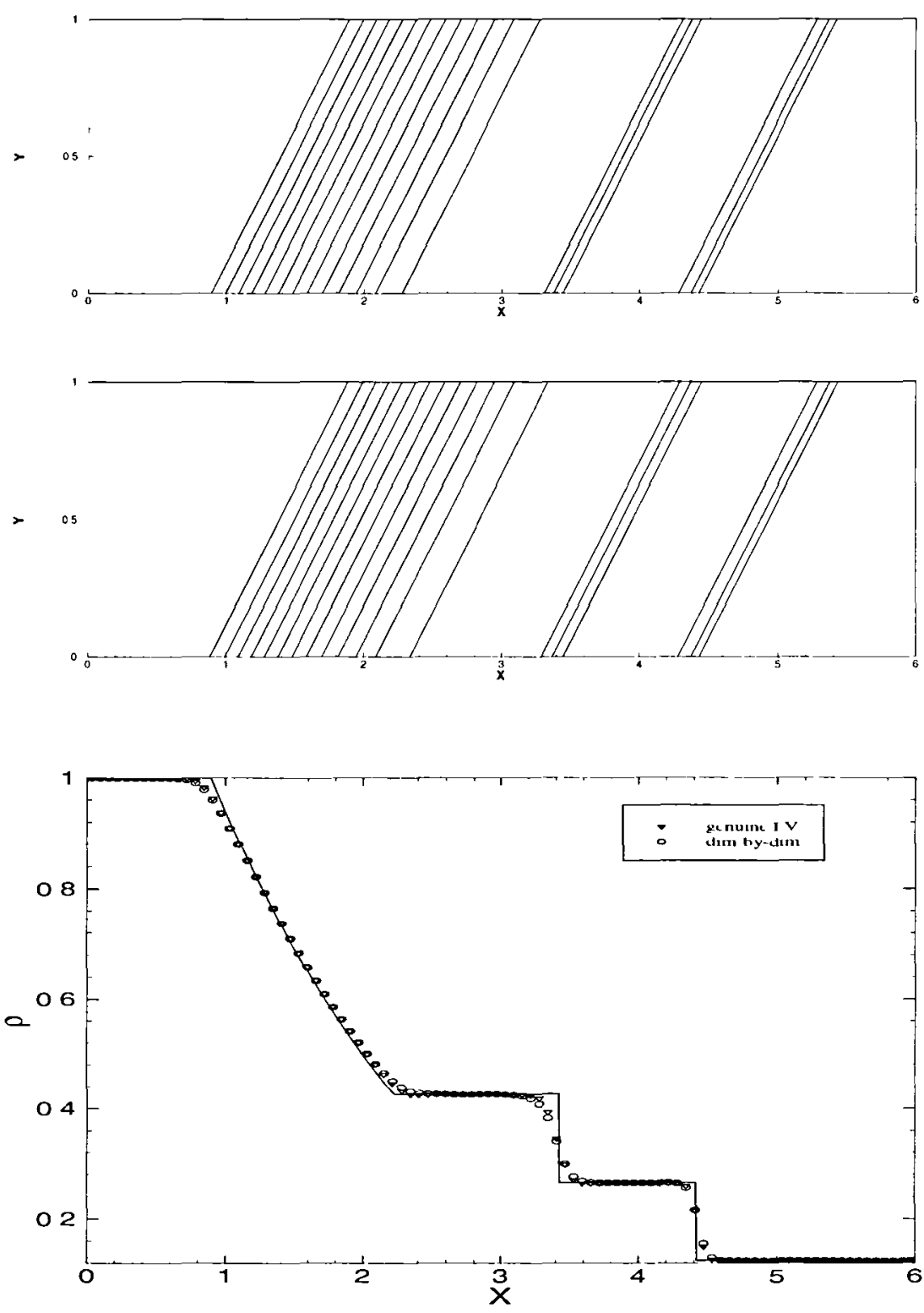

FIG 31 Oblique Sod's problem Density $\rho$ Top contour, genuıne two dimensional WENO, middle contour, dimensıon by dimension WENO, bottom cut at the bottom of the computational domain, the solid line is the exact solution, the trangles are the genume two dimensional WENO results, and the circles are the dimension by dimension WENO results

and making a $\theta=\frac{\pi}{4}$ angle with the $x$ axis The solution is computed up to $t=12$ on a $96 \times 16$ uniform mesh In Fig 31 we plot the density contours computed by the above two WENO schemes and the density cut at the bottom of the computational domain We can see that both schemes perform equally well in resolving the waves The genuine two dimensional scheme gives a slightly better resolution in the contact discontınuity and the rarefaction wave

3.4. A Mach 3 wind tunnel with a step. This model problem is originally from [25] The setup of the problem is The wind tunnel is 1 length unit wide and 3 length units long The step is 02 length 

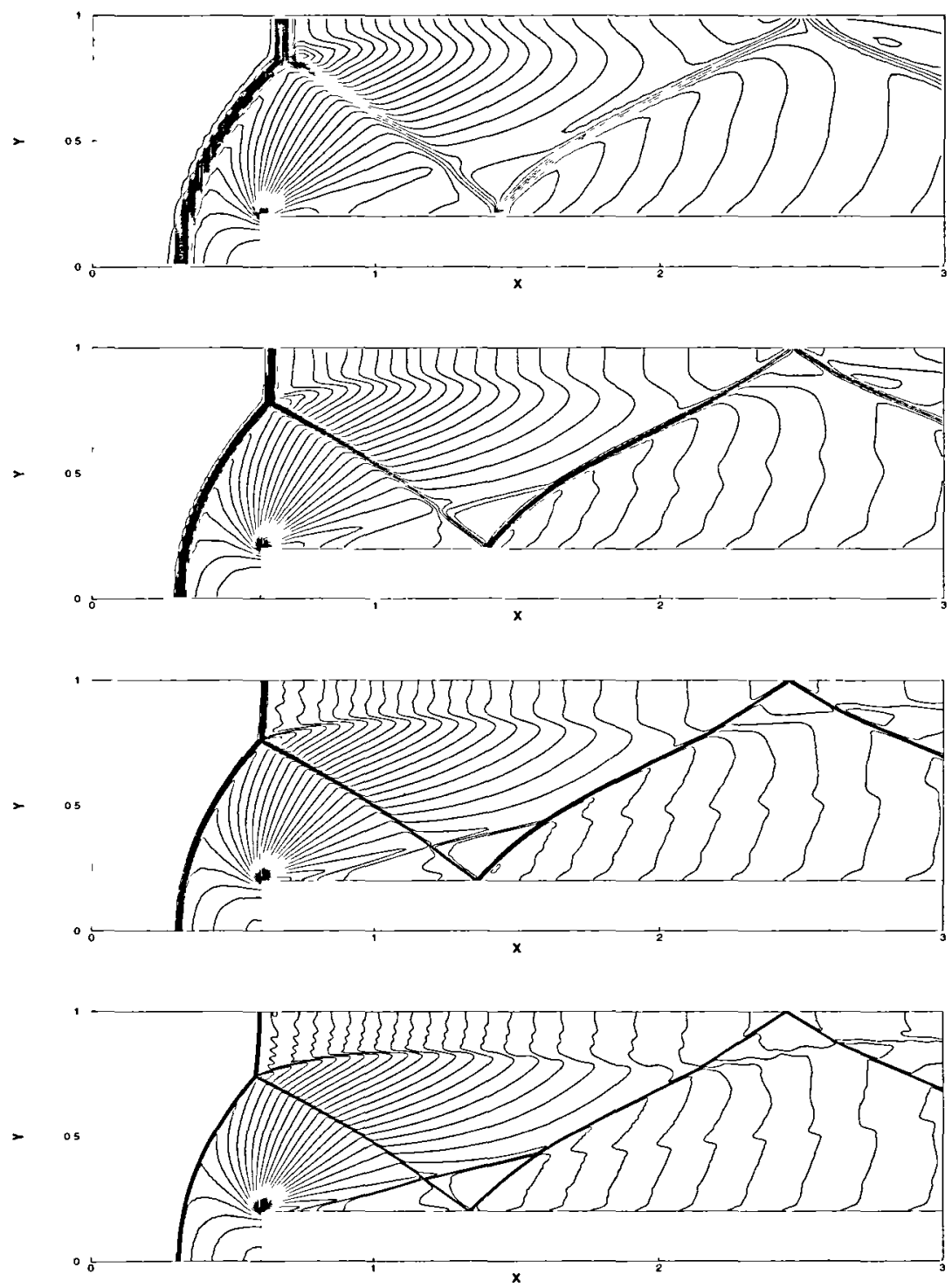

FIG 32 Forward step problem, $\Delta x=\Delta y=\frac{1}{40}, \frac{1}{80}, \frac{1}{160}, \frac{1}{320}$ from top to bottom 30 contours from 012 to 641 , dimension by dimension WENO

units high and is located 06 length units from the left-hand end of the tunnel The problem is initialized by a right-going Mach 3 flow Reflective boundary conditions are applied along the wall of the tunnel and inflow/outflow boundary conditions are applied at the entrance/exit The corner of the step is a singular point and we treat it the same way as in [25], which is based on the assumption of a nearly stcady flow in the region near the corner We show the density contours at time $t=4$ in Fig 32 Only the results from the dimension by dimension WENO scheme are shown Uniform meshes of $\Delta x=\Delta y=\frac{1}{40}, \frac{1}{80}, \frac{1}{160}, \frac{1}{320}$ are used

3.5. Double Mach reflection. This problem is also originally from [25] The computational domain for this problem is chosen to be $[0,4] \times[0,1]$ The reflecting wall lies at the bottom, starting from $x=\frac{1}{6}$ Initially a right-moving Mach 10 shock is positioned at $x=\frac{1}{6}, y=0$ and makes a $60^{\circ}$ angle with the $x$ axis 

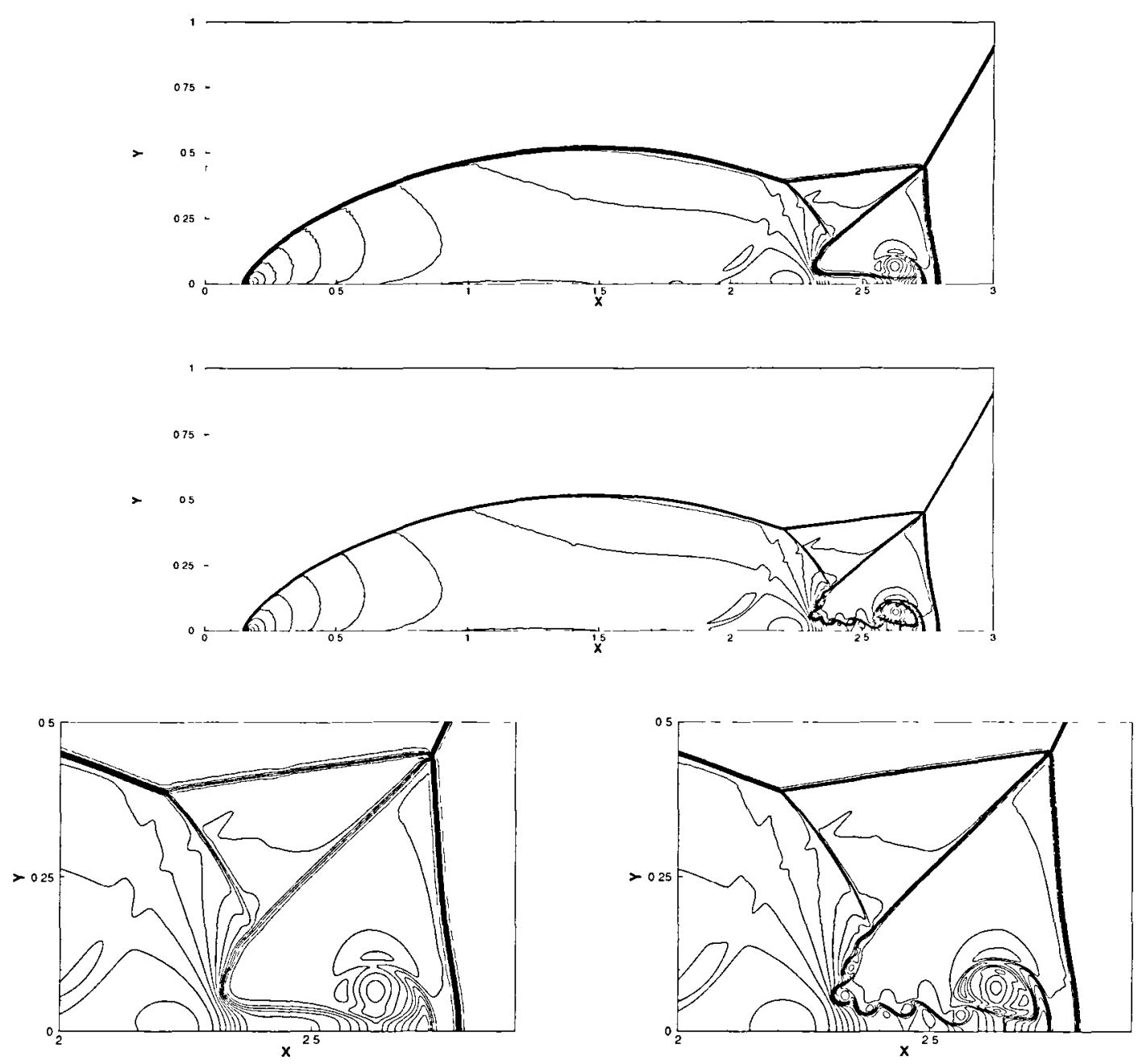

FIG 33 Double Mach reflection, $\Delta x=\Delta y=\frac{1}{240}$ (top and lower left) and $\frac{1}{480}$ (middle and lower right) Genuıne two dimensional WENO Blow-up regions at the bottom for detalls

For the bottom boundary, the exact post-shock condition is imposed for the part from $x=0$ to $x=\frac{1}{6}$ and a reflective boundary condition is used for the rest At the top boundary, the flow values are set to describe the exact motion of a Mach 10 shock We compute the solution up to $t=02$ Fig 33 and Fig 34 show the equally spaced 30 density contours from 15 to 227 computed by the genume two dimensional and the dimension by dimension WENO schemes We use uniform meshes with $\Delta x=\Delta y=\frac{1}{240}, \frac{1}{480}$ We can see that the results from both schemes are comparable

4. 2D finite volume WENO schemes on triangular meshes. Both third and fourth order finite volume WENO schemes on triangular meshes have been constructed in [8] The optional linear weights in such schemes are not unique These are then chosen to avold negative weights whenever possible, and if that falls, a grouping (of stencls) technique is used in [8], which works farrly well in the third order case with quite general triangulation but can yield positive weights for the fourth order case only with farly uniform triangulation In this section, we do not seek positive linear weights as in [8], but rather use the splitting technique to treat the negatıve linear weights when they appear For scalar equation, the scheme is stable 

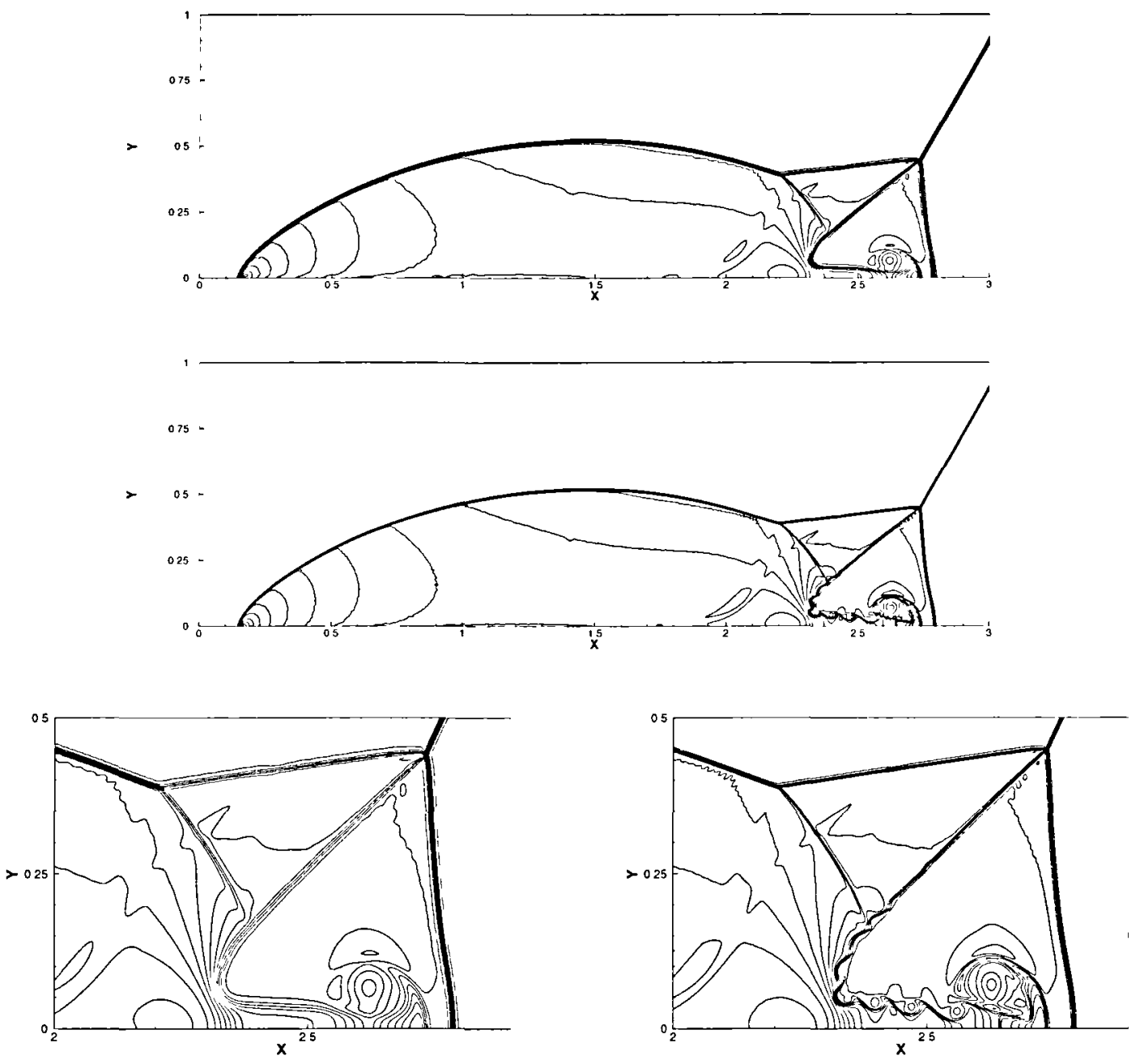

FIG 34 Double Mach reflection, $\Delta x=\Delta y=\frac{1}{240}$ (top and lower left) and $\frac{1}{480}$ (maddle and lower right) Dimensıon by dimension WENO Blow-up regions at the bottom for detals

in all runs For systems of conservation laws, there are still occasional cases of overshoot and instability, the reason seems to be related to characteristic decompositions and is still being investigated

4.1. Accuracy check for a smooth problem. We solve the 2D Burgers equation (1 10$)$ with the same initial and boundary conditions as before using the fourth order finite volume WENO scheme [8] The solution is computed up to $t=\frac{05}{\pi^{2}}$ when no shock has appeared The meshes used are 1) uniform meshes with equilateral triangulation and 2) random triangulation For the uniform meshes we do not seek positive weights as was done in [8], rather we use the splitting technique to treat the negative linear weights when they appear Table 41 indicates that close to fourth order accuracy can be achieved

4.2. Discontinuous problem 1: Scalar equation in 2D. Having shown the stable results $w 1$ th the splitting treatment of negative linear weights for a fourth order finite volume WENO scheme for the Burgers equation in section 2, we now test the fourth order WENO scheme on the Buckley-Leverett problem whose 
TABLE 41

$2 D$ Burgers equation accuracy check

\begin{tabular}{||c||c|c|||c||c|c||}
\hline \multicolumn{1}{||c||}{} & \multicolumn{3}{c||}{ uniform mesh } & \multicolumn{3}{c||}{ nonuniform mesh } \\
\hline \hline$\Delta x$ & $L^{\infty}$ error & order & $\Delta x$ & $L^{\infty}$ error & order \\
\hline $257 \mathrm{E}-1$ & $622 \mathrm{E}-4$ & & $267 \mathrm{E}-1$ & $211 \mathrm{E}-3$ & \\
\hline $129 \mathrm{E}-1$ & $461 \mathrm{E}-5$ & 375 & $126 \mathrm{E}-1$ & $235 \mathrm{E}-4$ & 292 \\
\hline $643 \mathrm{E}-2$ & $218 \mathrm{E}-6$ & 440 & $632 \mathrm{E}-2$ & $290 \mathrm{E}-5$ & 303 \\
\hline $321 \mathrm{E}-2$ & $138 \mathrm{E}-7$ & 398 & $334 \mathrm{E}-2$ & $261 \mathrm{E}-6$ & 378 \\
\hline $161 \mathrm{E}-2$ & $693 \mathrm{E}-9$ & 432 & $166 \mathrm{E}-2$ & $271 \mathrm{E}-7$ & 324 \\
\hline $808 \mathrm{E}-3$ & $670 \mathrm{E}-10$ & 340 & $744 \mathrm{E}-3$ & $157 \mathrm{E}-8$ & 355 \\
\hline
\end{tabular}

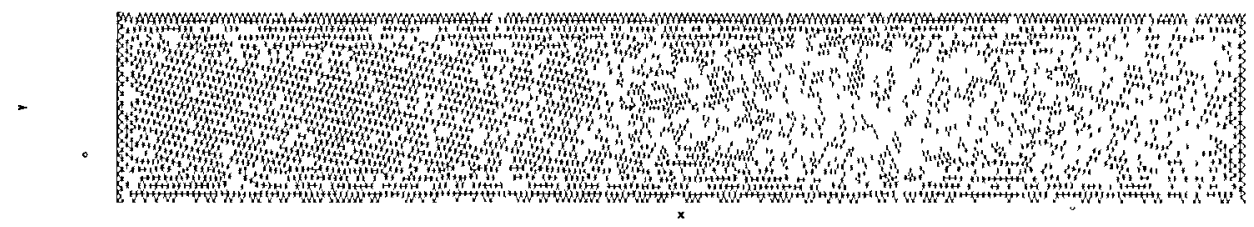

FIG $412 D$ Buckley-Leverett equation the mesh

flux is non-convex

$$
f(u)=\frac{u^{2}}{u^{2}+025(1-u)^{2}}, \quad g(u)=0
$$

with the initial data $u=1$ when $-\frac{1}{2} \leq x \leq 0$ and $u=0$ elsewhere The solution is computed up to $t=04$ The exact solution is a shock-rarefaction-contact discontinuity mixture The mesh we use here is a non-uniform triangulation, shown in Fig 41 Fig 42 shows that the waves have been resolved very well

4.3. Discontinuous problem 2: System of equations in 2D. We consider the 2D Euler equations in the domain $[-1,1] \times[0,02]$ The Sod and Lax shock tube initial data is set in the $x$ direction and periodic boundary condition is applied in the $y$ direction We use the fourth order finte volume scheme on triangular meshes to solve the above problem The mesh we use here is unfform But we do not seek positive weights as was done in [8], rather we use the splittıng technique in section 2 to treat the negative linear weights when they appear In fact we set delıberately certain linear weights to be negative to test the splitting technique Fig 43 shows the numerical results of the Sod and Lax problems

It seems that there are strll oscillations and instability for some non-uniform triangular meshes for the fourth order WENO schemes applied to Euler equations As the method works well for the same meshes with a scalar equation, the problem might be from the characteristic decompositions This is still under investigation

5. Concluding remarks. We have devised and tested a simple splitting technique to treat the negatıve linear weights in WENO schemes This technique involves very little additional CPU time and gives good results in most numerical tests The only case where it still yields oscillations and instability is when a fourth order finite volume WENO method is used on some non-uniform triangular meshes for Euler equations, the reason of which, presumably related to characteristic decompositions, is still under investıgation 

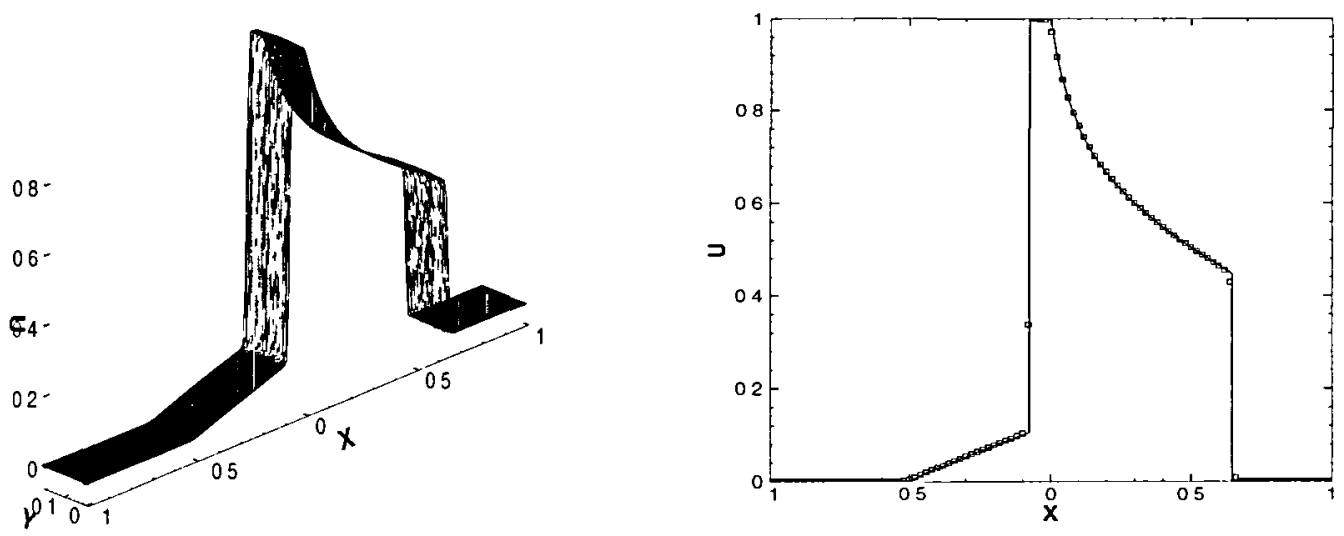

FIG $422 D$ Buckley-Leverett equation at $t=0$ 4, whth splatting Left the solution surface, Right the cut at $y=01$ (soltd line exact solution, symbols numerical solution)
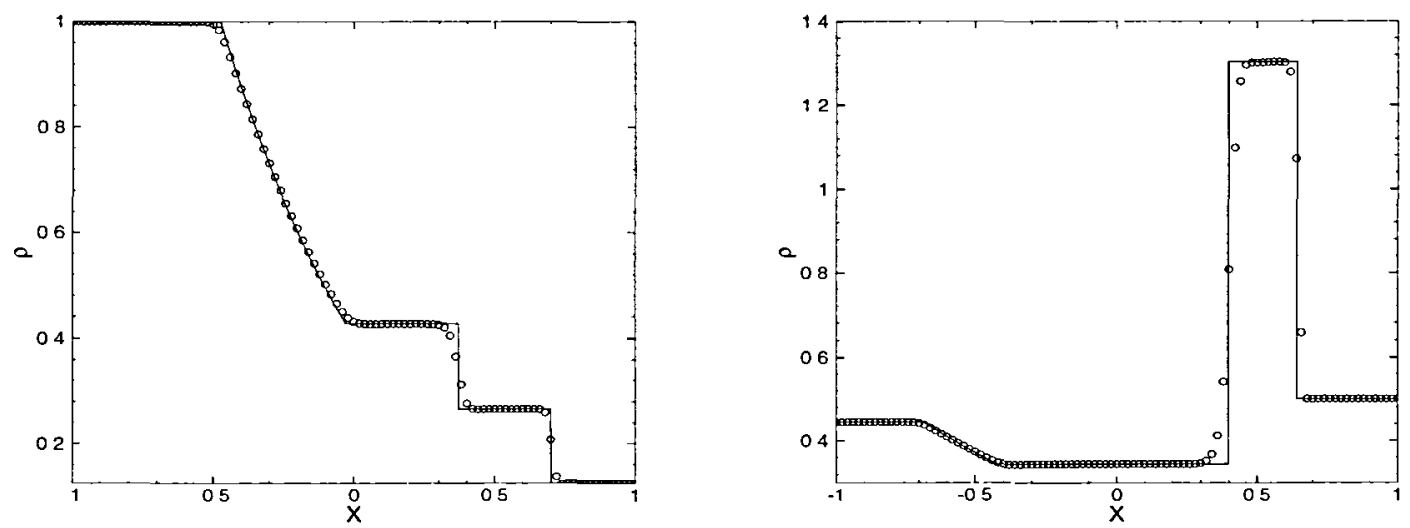

FIG 43 Density plot, Left Sod problem, Right Lax problem, with splitting Roughly 100 points in the $x$ direction

\section{REFERENCES}

[1] D BaLSARA AND C -W ShU, Monotonicity preserving weighted essentially non-oscillatory schemes with increasingly high order of accuracy, Journal of Computational Physics, 160 (2000), pp 405-452

[2] J Casper, C -W Shu and H L Atkins, Comparison of two formulations for high-order accurate essentıally nonoscillatory schemes, AIAA Journal, 32 (1994), pp 1970-1977

[3] L Del Zanna, M Velli And P Londrillo, Dynamical response of a stellar atmosphere to pressure perturbations numerical simulations, Astron Astrophys, 330 (1998), pp L13-L16

[4] O FRIEDRICHS, Werghted essentially non-oscillatory schemes for the interpolation of mean values on unstructured grids, Journal of Computational Physics, 144 (1998), pp 194-212

[5] F Grasso and S Pirozzoli, Shock-wave-vortex interactions Shock and vortex deformations, and sound production, Theor Comp Flu1d Dyn, 13 (2000), pp 421-456

[6] F Grasso and S Pirozzoli, Shock wave-thermal inhomogeneity interactıons Analysis and numerical simulations of sound generation, Phys Fluids, 12 (2000), pp 205-219 
[7] A Harten, B Engquist, S Osher and S Chakravarthy, Uniformly high order essentzally nonoscillatory schemes, III, Journal of Computational Physıcs, 71 (1987), pp 231-303

[8] C Hu AND C -W Shu, Werghted Essentially Non-Oscallatory Schemes on Trangular Meshes, Journal of Computational Physics, 150 (1999), pp 97-127

[9] G JiAng AND D -P PENG, Weighted ENO schemes for Hamilton-Jacobı equatıons, SIAM Journal on Scientific Computing, v21 (2000), pp 2126-2143

[10] G JiAng AND C -W SHu, Efficient implementation of weighted ENO schemes, Journal of Computational Physics, 126 (1996), pp 202-228

[11] G JIANG AND C -C WU, A high order WENO finite difference scheme for the equatıons of adeal magnetohydrodynamıcs, Journal of Computational Physics, 150 (1999), pp 561-594

[12] D Levy, G Puppo And G Russo, Central WENO schemes for hyperbolvc systems of conservation laws, Mathematıcal Modelling and Numerical Analysis $\left(M^{2} A N\right), 33$ (1999), pp 547-571

[13] D Levy, G Puppo And G Russo, Compact central WENO schemes for multudimensional conservatıon laws, SIAM Journal on Scientıfic Computing, 22 (2000), pp 656-672

[14] D Levy, G Puppo And G Russo, A thrrd order central WENO scheme for $2 D$ conservation laws, Appl Numer Math 33 (2000), pp 415-421

[15] S LiANG AND H ChEN, Numerical simulation of underwater blast-wave focusing using a high-order scheme, AIAA J , 37 (1999), pp 1010-1013

[16] R Liska ANd B Wendroff, Composite schemes for conservatıon laws, SIAM J Numer Anal, 35 (1998), pp 2250-2271

[17] R LiSKa AND B Wendroff, Two-dimensional shallow water equations by composite schemes, Int J Numer Meth Fl, 30 (1999), pp 461-479

[18] X -D LIU, S Osher AND T ChAN, Werghted essentially non-oscillatory schemes, Journal of Computational Physics, 115 (1994), pp 200-212

[19] P Montarnal AND C -W ShU, Real gas computation using an energy relaxation method and high order WENO schemes, Journal of Computational Physics, 148 (1999), pp 59-80

[20] S NOELLE, The MoT-ICE a new high-resolution wave-propagation algorthm for multıdimensional systems of conservation laws based on Fey's method of transport, Journal of Computational Physics, 164 (2000), pp 283-334

[21] C -W SHU, Essentıally non-oscillatory and werghted essentıally non-oscillatory schemes for hyperbolzc conservation laws, in Advanced Numerical Approximation of Nonlinear Hyperbolic Equatıons, B Cockburn, C Johnson, C-W Shu and E Tadmor (Editor A Quarteron), Lecture Notes in Mathematıcs, volume 1697, Springer, 1998, pp 325-432

[22] C -W SHu, High order ENO and WENO schemes for computational fluıd dynamics, in High-Order Methods for Computational Physics, T J Barth and H Deconinck, editors, Lecture Notes in Computational Science and Engineering, volume 9, Springer, 1999, pp 439-582

[23] C -W SHU AND S OSHER, Efficient implementation of essentially non-oscillatory shock capturing schemes, Journal of Computational Physics, 77 (1988), pp 439-471

[24] C -W SHU AND S OSHER, Efficient implementation of essentially non-oscillatory shock capturing schemes, II, Journal of Computational Physics, 83 (1989), pp 32-78

[25] P Woodward and P Colella, The numerical simulation of two-dimensional fluid flow with strong shocks, Journal of Computational Physics, 54 (1984) pp 115-173

[26] J YANG, S YANG, Y ChEn AND C Hsu, Implicit weighted ENO schemes for the three-dimensional 


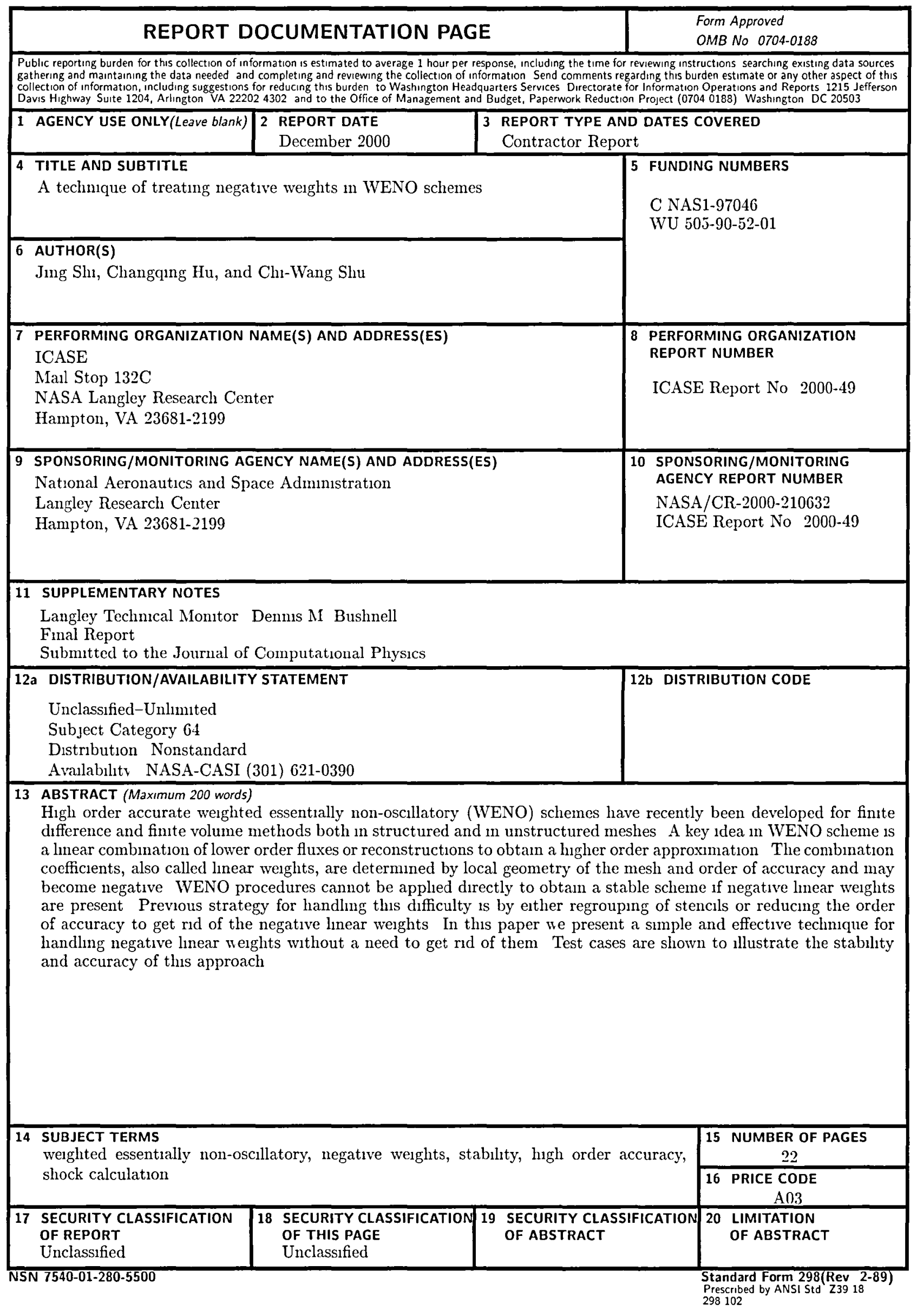


incompressible Navier-Stokes equatıons, Journal of Computatıonal Physıcs, 146 (1998), pp 464-487 


\section{End of Document}

